\title{
In-Situ Exploration of Objects on Oort Cloud Comet Orbits: OCCs, Manxes, ISOs
}

\section{Lead Authors:}

Karen J. Meech, University of Hawai'i, Institute for Astronomy, meech@ifa.hawaii.edu

Julie Castillo-Rogez, Jet Propulsion Laboratory, julie.c.castillo@jpl.nasa.gov

\section{Co-Authors/Endorsers:}

Erica Bufanda (University of Hawai'i, Institute for Astronomy), Marc Buie (SwRI), Olivier Hainaut (European Southern Observatory), Hope Ishii (University of Hawai'i, Hawaii Institute of Geophysics and Planetology), Jacqueline V Keane (University of Hawai'i, Institute for Astronomy), Jan Kleyna (University of Hawai'i, Institute for Astronomy), Jian-Yang Li (PSI), Sean Raymond (Laboratoire d'Astrophysique de Bordeaux), Richard Wainscoat (University of Hawai'i, Institute for Astronomy), Robert Weryk (University of Hawai'i, Institute for Astronomy), Bin Yang (European Southern Observatory) 


\section{Abstract}

NASA's competed mission calls are not compatible with missions that are responsive to new discoveries, i.e., missions that do not have a target at the time they are proposed. Relevant targets encompass Near Earth Objects that are potentially hazardous asteroids, Oort Cloud comets (OCCs, including Manx comets) and interstellar objects (ISOs). These objects represent the most primitive remnants of the processes that occurred during the formation of our solar system and other planetary systems and should be high priority for exploration. Two approaches have been suggested to explore these targets: spacecraft in storage, ready to launch following target discovery and spacecraft in standby orbit, as is being done by ESA's recently selected Comet Interceptor mission. In most cases the target is unknown at the time of spacecraft development, which bears a number of implications regarding the definition of basic spacecraft capability (e.g., delta-V) and payload. Among the three classes targets, the ISOs are expected to be the most diverse in terms of physical properties and outgassing activity. ISOs and Oort Cloud comets are challenging targets in terms of their orbital properties and fast velocities. Both mission scenarios have pros and cons. Launch following discovery offers greater flexibility in terms of target access but requires the fast turnaround of a launch vehicle. A spacecraft in a standby orbit is more responsive but has a more limited target accessibility. This white paper will provide suggestions and recommendations for broadening the SIMPLEx and Discovery calls so that they encompass reactive missions.

\section{The Science Case for Exploration}

Small primitive bodies witnesses the solar system's formative processes, including the contribution of presolar and interstellar sources. When gas was present in our solar system's protoplanetary disk, during the first 4-5 million years, a local chemical signature was imprinted on the planetesimals. The connection to today's solar system relies on how this material was dynamically redistributed during the planet-forming process. To connect early planet formation to the modern era, we must measure the compositions of a range of primitive bodies from different locations in the solar system and compare them with the predictions from models of early solar system formation, some of which predict significant reshuffling of material throughout the solar system. Long period comets (LPCs) are among the most difficult minor bodies to characterize due to their brief "oncein-a-human-lifetime" passages through the inner solar system. LPCs are typically brighter than short period comets because they have volatiles that turn on at larger distances. However, their activity also makes it very difficult to characterize their nuclei, and LPCs are rarely discovered before they are active. Large all sky surveys are changing this and LPCs are being discovered at very large distances, some even before the activity begins. A new class of objects on LPC orbits has been discovered with the Pan-STARRS survey that are nearly or completely inactive. Informally termed "Manxes" for their nearly tailless appearance, some have surface mineralogy that suggests similarity to inner solar system rocky material-i.e. they may have formed near the water-ice line (Meech et al., 2016). Even more recently, the first 2 interstellar objects have been discovered within our solar system: 1I/'Oumuamua, discovered by the PanSTARRS survey (Meech et al., 2017a) and 2I/Borisov, discovered by amateur astronomer G. Borisov. Like their counterparts within our solar system, these remnants of the process of planet formation hold the keys to understanding how solar systems form from their protoplanetary clouds - a key part of NASA's strategic goal. 


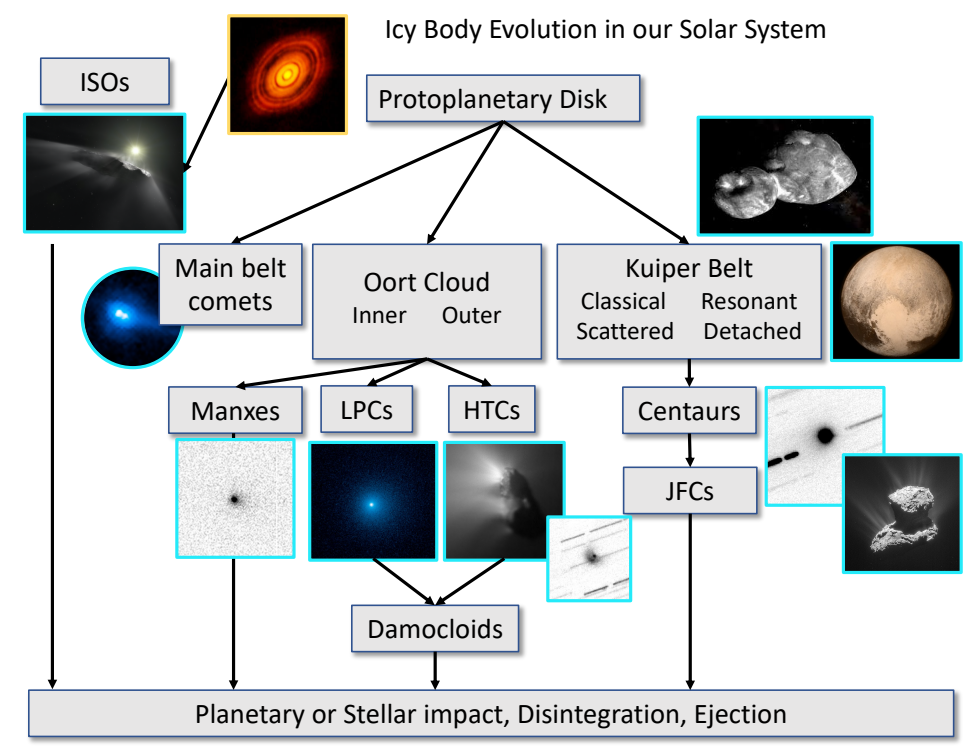

Figure 1: Genetic relationships of early solar system planetesimals. The Oort cloud is the largest reservoir of objects that has not been explored with an in situ mission. HTC $=$ Halley Type comet, $J F C=$ Jupiter Family Comet, ISO = Interstellar Object, arriving from another solar system.)

\subsection{Oort Cloud Comets}

LPCs come from the Oort Cloud (OC) — a swarm of relict planetesimals located between 5,000$100,000 \mathrm{au}$, emplaced during solar system formation-gravitationally scattered outward by the giant planets (mainly Jupiter; (Duncan et al., 1987; Dones et al., 2015). This happened after the Sun left its birth cluster (Tremaine, 1993; Pfalzner \& Bannister, 2019). Some fraction of the Oort cloud could have been captured from other stars in the Sun's birth cluster, if most stars scatter out a lot of planetesimals (Levison et al. 2010). OCCs are mostly pristine since they have not been repeatedly exposed to high insolation, in contrast to Halley Type (HTC) and Jupiter Family comets (JFC) which may have been significantly altered since entering the inner solar system. This is illustrated by the much higher level of activity for OCCs and their denser comae (Meech \& Svoren, 2004). Many OCCs are dynamically new with semi-major axes $>10^{4}$ au, i.e., recently injected into an inner solar system trajectory, never having entered the inner solar system. Some OCCs get perturbed onto hyperbolic orbits that lead to their escape from the solar system where they become interstellar objects (ISOs) in exoplanetary systems.

Encountering a pristine OCC, with near-surface ices and isotopic markers preserved from the solar system's formation would address many key questions identified in the Planetary Science Decadal Survey (Vision and Voyages), from understanding the initial stages of planet formation, to the origin of water, and planet migration and thus being responsive to NASA's strategic goal to understand "How did our solar system form and evolve?" Specific questions:

Q1. How did the solar system form from the protoplanetary cloud?

Q2. What is the history of solar system volatile and organic compounds?

Q3. What was the role of comets in the delivery of water to the inner solar system?

Q4. How is comet chemistry related to formation location or evolutionary processing history?

Q5. What is the cometary physical structure and how does this relate comet activity mechanisms? 


\subsection{Manx Comets}

Many dynamical models predict wide-scale planetesimal scattering during solar system formation (Raymond \& Izidoro, 2017) and thus ths source region of the Oort cloud comets is thought to encompass the entire outer Solar System (Brasser \& Morbidelli, 2013). On 2014 Sep. 22 the PanSTARRS survey discovered a weakly active comet (C/2014 S3) near its 2.0 au perihelion on a LP comet orbit. This could have been one of Oort's inactive LPCs that had lost it's "volatile frosting" (Oort, 1950). However, spectra showed that C/2014 S3's spectrum had the $1.0 \mu \mathrm{m}$ absorption characteristic of inner solar system rocky material; this represented something new. The activity was consistent with ice-driven sublimation, yet the minerals causing the $1 \mu \mathrm{m}$ absorption cannot form in the presence of water. This suggests that this tailless (or "Manx") object may have formed near the water-ice snowline and was ejected to the Oort cloud. In otherwords, we may have been looking at material that formed near the Earth-feeding zone. Characterization of these objects as a class may help distinguish between dynamical models of solar system formation.

\subsection{Interstellar Comets}

The recent discovery of the first interstellar object 1I/'Oumuamua passing through the solar system in 2017 has provoked intense, sustained interest by the scientific community. 'Oumuamua was accessible to ground based telescopes for less than a month, and a little longer from space. After this brief period of observation, 'Oumuamua's characteristics were quite different from what was expected from the first interstellar object (ISO), namely the first ISO was expected to have obvious cometary activity. Over 120 papers have been written about this object (and this number continues to grow). Incorporating a diverse range of scientific disciplines including galactic, stellar, and planetary dynamics, planetesimal formation, tidal disruption, shape modeling, and the nature and evolution of comets, this one discovery has energized a new interdisciplinary awareness in the study of planet formation. ISOs enable the close up study of material from other planetary systems, allowing us to assess similarities and differences in the chemistry and physical processes driving planetary growth in other planetary systems. The second ISO, 2I/Borisov, was discovered less than 2 years after the first, much sooner than expected, and has characteristics which are very different from 'Oumuamua. When LSST comes on line, it will greatly increase the discovery rate.

Highlighting exploration of objects on LPC orbits-OCCs, Manxes, and ISOs (one of the last unexplored classes in the solar system), in the next decadal survey's New Frontiers program is the natural next step in the long term roadmap for deciphering solar system origins contained in comets. It is the logical and necessary mission after the recent exploration of comet $67 P$ by the Rosetta mission and its possible sampling by the CAESAR mission in the future.

\section{Goals and Objectives of OCC Exploration}

Recent models of Solar System formation show that small icy planetesimals have had a rich dynamical history, suggesting that the chemical fingerprints of formation within the solar nebula cannot be fully interpreted without coupled dynamical models. Thus, by being selective in our choice of targets for NASA's missions to comets, we could potentially map the changing chemistry of the condensible fraction of the nebula with relatively few missions (e.g., one mission to a comet formed primarily from interstellar materials with high $\mathrm{CO}, \mathrm{N}_{2}$, and amorphous dust, one mission to a comet formed near the end of nebular accretion with high $\mathrm{C}_{2} \mathrm{H}_{6}$ and other hydrocarbons plus 
$\mathrm{NH}_{3}$ and highly crystalline dust, and another mission to a comet whose composition appears to be intermediate relative to the previous two)." The specific science objectives that could be addressed through in situ exploration of these objects and how they address the Decadal goals are:

1. Determine the nature, abundance, and formation temperature of cometary volatiles (Q1-4)

2. Determine the nature, abundance, and origin of cometary solid grains (Q1, Q4)

3. Track outgassing activity over a range of distances $(\mathrm{Q} 2, \mathrm{Q} 5)$

4. Characterize the physical properties of the nucleus (Q5)

\subsection{Technical Approach and Methodology}

These objectives directly respond to NASA's strategic goals to understand "How did our solar system form and evolve?" with flow down to science goals framed by NASA's Planetary Science Division to "Explore and observe the objects in the solar system to understand how they formed and evolve" and "Advance the understanding of how the chemical and physical processes in our solar system operate, interact and evolve".

Objective 1 - Current state: OCCs are more active than JFCs, but we don't know to what extent this is related to formation or evolution (Meech, 2017). There is now a strong indication that there were different chemical reservoirs in the disk that were not directly related to current dynamical classifications (A'Hearn et al., 1995; Mumma \& Charnley, 2011). We have a handful of comets for which $\mathrm{D} / \mathrm{H}$, Oxygen isotopes and Nitrogen isotopes have been measured but have only one comet for which noble gases have been measured (67P, Rubin et al., 2018), and this can provide important information about the temperature at which the comet formed. Carbonaceous chondrite meteorites (representative of C-type asteroids) match the Earth's water and N isotopes (Marty, 2012). Some comets match Earth's D/H but none match its $\mathrm{N}$ isotopes (Marty, 2012). Measurements of these isotopes for an $\mathrm{OCC}$ would allow for comparison with other small body classes. The most dramatic outcome would be if an OCC was like a carbonaceous chondrite but with more water. That would indicate a common origin with C-type asteroids and Earth's water. Current dynamical models suggest that carbonaceous material likely formed beyond the growing Jupiter and was scattered by the growing/migrating giant planets and implanted into the inner Solar System (Raymond \& Izidoro, 2017; Desch et al., 2018). Alternately, if its $\mathrm{D} / \mathrm{H}$ and other isotopic ratios are very different it would indicate that the comet did not come from the same reservoir as C-types and Earth's water. Regardless, these measurements will constrain the object's source distribution within the Solar System, which would feed back to dynamical models. These measurements require an in-situ mission. Investigations: Ultraviolet and near-infrared spectroscopy; mass spectroscopy of noble gases and other volatiles, particularly targeting isotopic markers; sub-millimeter wave spectroscopy.

Objective 2 - Current state: Observations from Giotto/VEGA, Spitzer, Stardust, Rosetta, interplanetary dust particles, and micrometeorites have shown that cometary dust exhibits considerable diversity between comets and within comets at micron scales. The dust is composed of a wide range of materials from amorphous silicates and refractory organics that may be of interstellar and/or early Solar System heritage, to high temperature minerals that must have been mixed outwards from the inner solar nebula, and even materials that have seen aqueous alteration in parent bodies (Wooden et al., 2017). This diversity of materials has important but still poorly understood implications for dust thermal processing and transport in the protoplanetary disk. A key Rosetta mission finding is that organic molecules containing abundant hydroxl groups and refractory macromolecular material bearing aliphatic and aromatic hydrocarbons make up $50 \%$ of the 
dust. Investigations: Elemental and selected isotopic compositions of dust with mass spectroscopy; Characterization of functional groups in NIR spectroscopy.

Objective 3 - Current state: Water ice sublimation can drive activity out to 5-6 au. However, most OCCs are active at much larger heliocentric distances due to the sublimation of more volatile ices $\left(\mathrm{CO}\right.$ or $\left.\mathrm{CO}_{2}\right)$, or other processes (Meech et al., 2009). Due to lack of observations large heliocentric distances, and especially, of the onset of their activity, our knowledge of the processes driving the distant activity of comets remains incomplete. Following the Deep Impact and Rosetta missions it appears that small and short duration outbursts might be frequent in active comets, while much larger scale outbursts are also detected from ground-based observations (A'Hearn et al., 2005; Pajola et al., 2017). Understanding the activity of comets and the origin of outbursts is key to improve our knowledge of nucleus composition and structure. Investigations: Combined observations from ground-based assets, beginning immediately upon the OCC's discovery when it is beyond $10 \mathrm{au}$ and continuing until it is no longer detectable by the most powerful telescopes on Earth, and observations from the spacecraft before, during, and after flyby.

Objective 4 - Current state: Only 6 comet nuclei have been directly imaged and their physical characteristics are varied (Groussin et al. 2019). The nuclei are often bilobate and display complex surface geology, from pits to scarps and smooth terrains. It is unclear if these characteristics are primordial or evolved. OCC exploration would enable a unique view of a pristine, or significantly less-evolved, cometary nucleus. This would compliment exploration of the Kuiper Belt by New Horizons, which explores a different class of small bodies, originating from a different region of the protoplanetary disk (Stern et al., 2019). Nucleus Investigations: Visible imaging to characterize the shape, rotation, and global geology; Map color and albedo variations to constrain surface composition; characterization of rotation state; characterize thermal properties (temperature, thermal inertia); IR spectroscopy to identify surface ices / mineralogy.

\subsubsection{Science-Driven mission Requirements and Challenges}

Exploring the Right Target: In order to investigate the primordial chemistry of the protoplanetary disk, we need to explore a dynamically new OCC, undergoing its first pass near the Sun. It is plausible such a target would also be a "Great" comet, i.e., a comet that is $>10 \mathrm{~km}$ in size, such as $\mathrm{C} / 1995 \mathrm{O} 1$ (Hale Bopp) or C/2017 K2. The latter was discovered while it was $>15$ au from the Sun owing to its already significant activity and $10^{5} \mathrm{~km}$ scale coma (Meech et al., 2017b). These objects have proved exceptional but happen at least once in a decade. Manxes and ISOs appear to be discovered more frequently.

Close Approach Operations near Perihelion: The above investigations require proximity operations and crossing the dust tail when the outgassing is fully developed. This is driven by the need to sample the comet's rich volatile inventory. The obvious drawback is that the cometary environment is hazardous (comet Hale Bopp's dust production was $\sim 10^{6} \mathrm{~kg}$ per second at its perihelion). These operations will require the spacecraft to carry a Whipple shield and we will explore the latest developments in that area. Nucleus density determination is only possible for a Manx or nearly inactive ISO, because gravity science requires a very close flyby (i.e., a few kilometers). Intense outgassing activity will likely preclude close proximity measurements. Non-gravitational forces on the comet may enable indirect constraints on the bulk density.

Multiple and Potentially Conflicting Investigations in Short Window: Encountering the target at perihelion implies relative encounter velocities $>30 \mathrm{~km} / \mathrm{s}$. Although the comet's coma is expected 
to expand over $>10^{6} \mathrm{~km}$, many science operations will need to be accomplished in a very short time and in various locations (nucleus, inner coma, dust tail, plasma tail), under different and potentially conflicting vantage points. Hence, the mission concept needs to identify strategies to optimize science return under challenging conditions and limited lifetime.

Material Sampling at $10 \mathrm{~s} \mathrm{~km} / \mathrm{s}$ : An important focus of the proposed mission concept will be sampling volatiles for isotopic measurements. A sample return "a la Stardust" would be ideal but comes with major challenges. Material capture in aerogel was already complex in the case of Stardust and led to the loss of organics and the degradation of other fragile species (JaramilloBotero et al., 2012). Similarly, organics are significantly degraded, or even destroyed, during impacts at velocities $>\sim 6 \mathrm{~km} / \mathrm{s}$ into mass spectrometers (MS). At the much faster velocities considered here, many compounds can react with the MS chamber, leading to ambiguous results (Brownlee, 2014). On the other hand, dust spectroscopy is particularly suited for investigating the products of hypervelocity impacts. With an instrument such as the SUrface Dust Analyser (SUDA) or a derivative, material destroyed upon impact is analyzed with a MS that yields elemental ratios and organic functional groups (Horanyi, 2019).

\subsection{Challenges and Exploration Strategies}

\subsubsection{Dealing with a Challenging Target}

Oort cloud comets are "once in a lifetime" targets. They have orbital periods ranging from thousands to $>1 \mathrm{My}$. Their exploration is challenging for four main reasons: their orbital properties are unknown in advance but they frequently have high inclinations (Figure 2A); the encounter speeds are typically high (10s of $\mathrm{km} / \mathrm{s})$, hence the encounters may be very short; their perihelion may be at several au from the Sun; and there may be risks if the object ejects dust at high velocity.
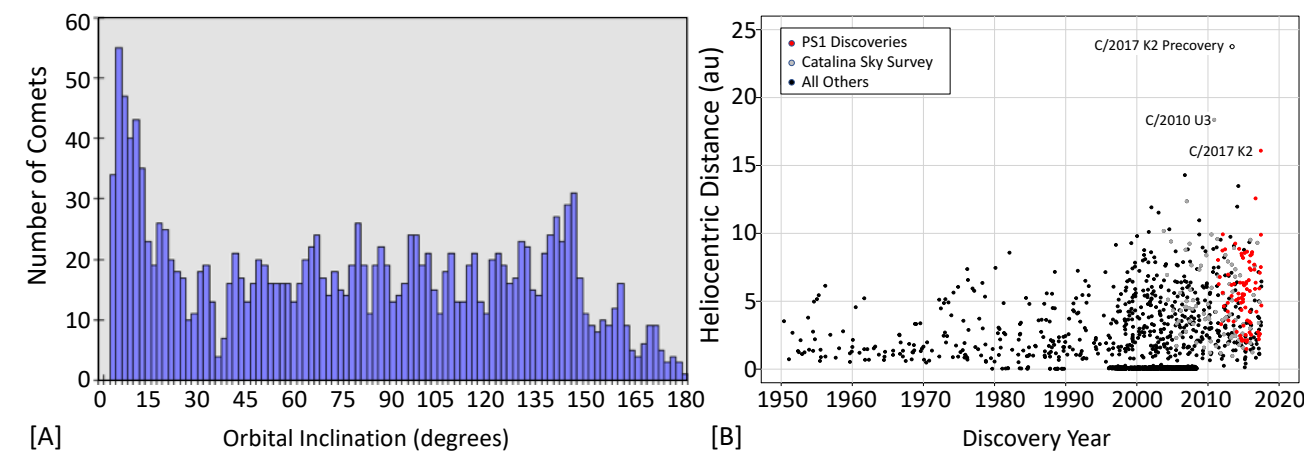

Figure 2: [A] Orbital inclination distribution of observed long-period comets (Willman Jr 1995). [B] Pre-perihelion comet discoveries show that the introduction of the Pan-STARRS and Catalina Sky Surveys changed the game by enabling the detection of large OCCs years before their perihelia.

The only attempt to explore a non-JFC up close was the 1986 encounter of comet 1P/Halley at a relative velocity of $\sim 79 \mathrm{~km} / \mathrm{s}$. Its visit was deemed such an important event that six spacecraft were sent by different space agencies: NASA, ESA, Roskosmos (USSR), and JAXA (its first space mission). The missions were mostly uncoordinated and the proposed science was only partially realized because the violence of the dense dust environment destroyed some of the instruments. This is the only time six spacecraft have been deployed, and while bold in concept, there was only modest science return in comparison to the level of resources engaged. Future mission architecture should take advantage of small constellations to mitigate risk for these high-science return targets. 


\subsubsection{Exploration Strategies}

It is expected that existing (Pan-STARRS) and upcoming (LSST) surveys will allow the discovery of OCCs more than 5 years before perihelion. For example, two recent "Great" comets were discovered $>15$ years before perihelion (Figure 2B). C/2017 K2 (PANSTARRS) was discovered 5.5 years before perihelion but the Sloan Digital Sky Survey image archive revealed that it was visible in 2013 at $23 \mathrm{au}$ - almost ten years before perihelion (Meech et al., 2017b).

Hence, the exploration of OCCs may require a proactive approach: preparing a spacecraft to be ready for launch (either on the ground or from a standby orbit) in anticipation of target discovery. While this approach breaks the current mission implementation paradigm, it is aligned with interest from the planetary science community for reactive missions, e.g., for NEO exploration, planetary defense, and for future interstellar objects crossing our solar system. Please also see the follow-on white paper (August deadline) where we will address strategies for proposing reactive missions.

\section{References}

A'Hearn, M. F., Millis, R. C., Schleicher, D. O., Osip, D. J., \& Birch, P. V. 1995, Icarus, 118, 223

A'Hearn, M. F., Belton, M. J. S., Delamere, W. A., et al. 2005, Science, 310, 258

Brasser, R., \& Morbidelli, A. 2013, Icarus, 225, 40

Brownlee, D. 2014, Annual Review of Earth and Planetary Sciences, 42, 179

Desch, S. J., Kalyaan, A., \& O’D. Alexander, C. M. 2018, , 238, 11

Dones, L., Brasser, R., Kaib, N., \& Rickman, H. 2015, Space Sci. Rev., 197, 191

Duncan, M., Quinn, T., \& Tremaine, S. 1987, AJ, 94, 1330

Groussin, O., Attree, N., Brouet, Y., et al. 2019, Space Sci. Rev., 215, 29

Horanyi, M., e. a. 2019, in IEEE Aerospace Conference Proceedings

Jaramillo-Botero, A., An, Q., Cheng, M.-J., et al. 2012, Physical Review Letters, 109, 213201

Levison, H. F., Duncan, M. J., Brasser, R., \& Kaufmann, D. E. 2010, Science, 329, 187

Marty, B. 2012, Earth and Planetary Science Letters, 313, 56

Meech, K. J. 2017, Phil. Trans. of the Royal Society of London Series A, 375, 20160247

Meech, K. J., \& Svoren, J. 2004, in Comets II, ed. M. Festou, H. Keller, \& H. Weaver, 317

Meech, K. J., Pittichová, J., Bar-Nun, A., et al. 2009, Icarus, 201, 719

Meech, K. J., Yang, B., Kleyna, J., et al. 2016, Science Advances, 2, e1600038

Meech, K. J., Weryk, R., Micheli, M., et al. 2017a, Nature, 552, 378

Meech, K. J., Kleyna, J. T., Hainaut, O., et al. 2017b, ApJ, 849, L8

Mumma, M. J., \& Charnley, S. B. 2011, ARA\&A, 49, 471

Oort, J. H. 1950, BAN, 11, 91

Pajola, M., Höfner, S., Vincent, J. B., et al. 2017, Nature Astronomy, 1, 0092

Pfalzner, S., \& Bannister, M. T. 2019, ApJ, 874, L34

Raymond, S. N., \& Izidoro, A. 2017, Icarus, 297, 134

Rubin, M., Altwegg, K., Balsiger, H., et al. 2018, Science Advances, 4, eaar6297

Stern, S. A., Weaver, H. A., Spencer, J. R., et al. 2019, Science, 364, aaw9771

Tremaine, S. 1993, in ASPCS, Vol. 36, Planets Around Pulsars, ed. J. Phillips, S. Thorsett, \& S. Kulkarni, 335-344

Willman Jr, A. J. 1995, Implications of Magnitude Distribution Comparisons between TNOs and Comets, http://www .princeton. edu/ willman/tno-comet/tnocomet.pdf, ,

Wooden, D. H., Ishii, H. A., \& Zolensky, M. E. 2017, Phil. RSTA, 375, 20160260 